\title{
Investigation of a side wall heated cavity by using lattice Boltzmann method
}

\author{
Ridha Djebali*,** — Mohammed El Ganaoui* \\ Habib Sammouda**
}

* CNRS/ SPCTS, UMR 6638, FST Limoges, Dép. of Physics, 87060 Limoges, France

ganaoui@unilim.fr

** University of Tunis El-Manar, FSMPN Tunis,

Dép. of Physics, LETTM, 1060 Tunis, Tunisia

\begin{abstract}
The lattice Boltzmann method based on the BGK model has been used to simulate laminar natural convection in a heated rectangular cavity on the uniform grid. The hydrodynamic and thermal fields are solved by using the double populations approach. A general benchmark has been carried out to show the effects of secondary parameters at their wide range. Excellent agreement is obtained by comparison with available literature.

RÉSUMÉ. La méthode de Boltzmann sur réseau basée sur l'approximation BGK a été employée pour la simulation de la convection naturelle laminaire au sein d'une cavité différentiellement chauffée. Le benchmark établi montre l'effet de divers paramètres secondaires pour de moyennes échelles. Un bon accord est démontré par comparaison avec des résultats de littérature.

KEYWORDS: lattice Boltzmann method, natural convection, laminar flow, benchmark solution, secondary parameters effects.

MOTS-CLÉS : méthode de Boltzmann sur réseau, convection naturelle, écoulement laminaire, solution benchmark, effets des paramètres secondaires.
\end{abstract}

DOI:10.3166/EJCM.18.217-238 @ 2009 Lavoisier, Paris 


\section{Introduction}

The lattice Boltzmann method (LBM) derives from the lattice Boltzmann equation and the result is a discrete distribution function which represents a probability of finding particles with a certain range of velocities at certain range of locations at a given time (Succi, 2001; Sukop et al., 2005; Dubois, 2006; Mohamad, 2007).

The LBE is a mesoscopic approach that presents many features. First, it is based on the kinetic equations and statistical physics, unlike of the conventional methods which are based on the continuum mechanics; second, it can recover the hydrodynamic behaviour at the macroscopic level (mass, momentum and energy conservation) in second order of accuracy in time and space; third, it is unconditionally stable $\left(C F L=e_{\alpha} . \delta t / \delta x=1\right.$ based on the lattice units $\left.\delta t=\delta x=1\right)$ and presents a linear form in its scheme (algebraic operations) which overcome CFL condition of stability and the drawback of the non-linear form of the Navier-Stokes equations (which leads generally to algebraic equations); forth, the particularity of local collision step goes with new technology of parallel computers.

For its efficiency and accuracy, the LBM has received a considerable attention in the earlier years by fluid dynamic researchers (Zou et al., 1997; Chen et al., 1998; Guo et al., 2000),. This new approach has proved the ability to simulate large variety of fluid flows (Mezrhab et al., 2007; Jami et al., 2007) and has expressed a flexibility and simplicity to simulate both 2D (Bouzidi et al., 2001) and 3D (D'Humières et al., 2001) and to handle complex geometries (Bouzidi et al., 2001; Lallemand et al., 2003).

The first investigations using LBM concern the hydrodynamic character. However, the thermal flows are almost always encountered in industrial applications. (Massaioli et al. 1993) attempt the first investigation of thermal LB model; they have developed first the thermal LBM and now there are three thermal LBM models. The multispeed approach (Chen et al., 1998), the passive scalar approach (D'Orazio et al., 2004) and the double population approach (He et al., 1998).

An extension of the passive-scalar thermal model, where viscous and compressive heating are neglected, was recently proposed by introducing the Internal Energy Density Distribution Function (IEDDF model). So, the omitting of the viscous heat dissipation and compression work done by pressure in macroscopic energy equation can be reflected by dropping out the gradient term in the evolution equation for the new distribution function, since such gradient term is mainly used to recover these terms through the Chapman-Enskog expansion (Hou et al., 1995). Following the work of (Peng et al., 2003) a simplified thermal distribution model is proposed. This model was proved to fully recover the energy equation at the macroscopic level incorporating work and heat dissipation correctly and particularly, it keeps the feature of standard LBM.

Now, the efficiency and the accuracy of the LBM reside on its ability to model complicated flows such as multiphase flow, chemically reacting flows, micro-flows 
in $2 \mathrm{D}$ and $3 \mathrm{D}$ isothermal and thermal flows on uniform and non uniform grid (Shu et al., 2002-2005) and (Semma et al., 2008).

Natural convection in heated square cavity (insulated from below and above, the vertical wall are maintained at a fixed temperature) was not only an ideal case for testing numerical models intended for solving Navier-Stokes equations, but also it is present in many fields such as that of aeronautics, electronics, heat transfer, in buildings and it concerns also the application of solar energy collectors and double glazed windows. In other way, it was shown in literature that several parameters can influence the dynamic and thermal behaviours within the heated cavity the effect of the Rayleigh number, as a monitoring parameter of the convection, was almost controlled and its values criticizes signs of transitions were encircled (de Gassowski et $a l ., 2003)$, heated square cavity. Various numerical simulations have been performed to study the major effects of the different parameters (Prandtl number, the orientation of the cavity and the aspect ratio $(A r=W / H$ : width/height $))$ on the structure of the flow, but few studies are conducted using LBM and also the coupled effects of these parameters are not examined and discussed by thermal LB models.

The purpose of this article is to validate the model through its application to the configuration of heated square cavity (Figure 1) and to establish a general benchmark reproducing the former results corresponding to the effect of each parameter in a wide range of the Rayleigh number values $\left(10^{3} \leq R a \leq 10^{6}\right)$, the Prandtl number $(0.025 \leq \operatorname{Pr} \leq 6)$, the inclination of the cavity from the horizontal plane $(0 \leq \gamma \leq 3 \pi / 4)$ and the aspect-ratio $(1 / 8 \leq A r \leq 8)$. The present study investigates also the mutual effects on the heat transfer expressed by the Nusselt number, some correlations will be therefore established. The fluid is assumed to be incompressible, the Boussinesq approximation stays valid and the radiation effects are neglected.

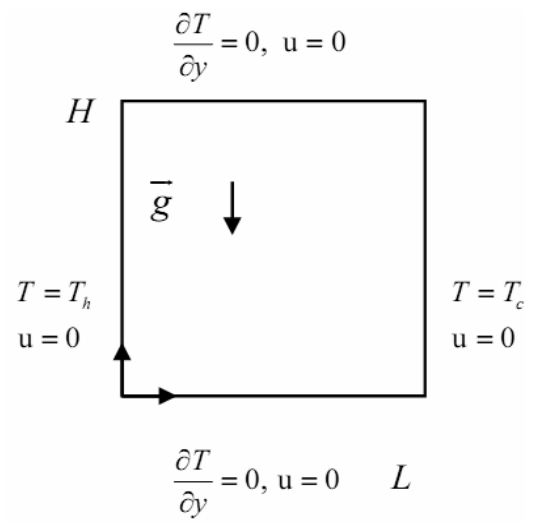

Figure 1. Natural convection problem in a square cavity 
The remaining part of the paper is organized as follow. Section 2 introduces a brief description of the lattice Boltzmann method in presence of buoyancy force. Dimensionless parameters and implementation of boundary conditions are also presented. Section 3 presents a validation of the model through its application to exhibit dynamic and thermal behavior in heated differentially air-filled square cavity. Order of accuracy of the model is demonstrated. In section 4 , the effects of a quite number of secondary parameters linked to fluid, configuration and orientation are studied separately at first and then coupled.

\section{Brief description of the simplified thermal lattice Boltzmann model}

\subsection{The thermal lattice Boltzmann model}

The well known Boltzmann equation takes the following form:

$$
\frac{\partial f}{\partial t}+\xi \cdot \nabla_{x} f+a \cdot \nabla_{\xi} f=\Omega(f, f)
$$

where $f=f(x, \xi, t), a$ and $\Omega$ are the single particle distribution function, the body force and the collision term. $x, \xi$ and $t$ denote position, velocity and time.

The complicated collision term makes the Boltzmann equation difficult to solve. After the popular BGK (Bhatnagar-Gross-Krook, 1954) and (Welander, 1954) approximations (Mohamad, 2007), which plays a major role in the theory of the lattice Boltzmann equation, the collision term is well simplified and takes the new form:

$$
\Omega_{B G K}=-\frac{1}{\lambda}\left(f-f^{e q}\right)
$$

where $\lambda$ is a typical time-scale associated with collisional relaxation to the local equilibrium.

The exploitable discrete form of the lattice Boltzmann equation, after introducing BGKW approximation, becomes:

$$
f_{\alpha}\left(x+e_{\alpha} \Delta t, t+\Delta t\right)-f_{\alpha}(x, t)=-\frac{1}{\tau_{v}}\left(f_{\alpha}(x, t)-f_{\alpha}^{e q}(x, t)\right)+e_{\alpha} \Delta t . F_{\alpha}
$$

where $F_{\alpha}$ is the external body force in the direction of the lattice discrete velocity $e_{\alpha}, \tau_{v}=\lambda / \Delta t$, and $f_{\alpha}^{e q}$ the equilibrium distribution function.

The philosophy of discrete velocity defines a terminology to the LB equation that is the lattices and arrangements noted as $D n Q m$. Here $n$ and $m$ represent the dimension of the problem and number of streaming direction. In the case of our two 
dimensional problem, the D2Q9 square lattice is used. Such model is characterized by nine discrete velocities defined as:

$$
e_{\alpha}= \begin{cases}(0,0) & \alpha=0 \\ (\cos [(\alpha-1) \pi / 2], \sin [(\alpha-1) \pi / 2]) & \alpha=1,2,3,4 \\ (\cos [(\alpha-5) \pi / 2+\pi / 4], \sin [(\alpha-5) \pi / 2+\pi / 4]) \sqrt{2} . c & \alpha=5,6,7,8\end{cases}
$$

and an equilibrium distribution function defined as:

$$
f_{\alpha}^{e q}=\omega_{\alpha} \rho\left[1+3 \frac{\vec{e}_{\alpha} \vec{V}}{c^{2}}+\frac{9}{2} \frac{\left(\vec{e}_{\alpha} \vec{V}\right)^{2}}{c^{4}}-\frac{3}{2} \frac{\vec{V}^{2}}{c^{4}}\right]
$$

where $w_{0}=4 / 9, w_{\alpha}=1 / 9$ for $\alpha=1,2,3,4 \quad w_{\alpha}=1 / 36$ for $\alpha=5,6,7,8$ are a weight factors, $c=\Delta x / \Delta t$ the lattice streaming velocity and $\vec{V}=(u, v)$.

The sketch of the nine-velocities model is shown in Figure 2.

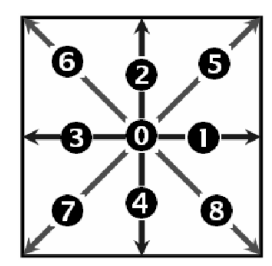

Figure 2. The nine-velocities LBM model on $2 D$ square lattice

The macroscopic fields, density and velocity, are computed using the zero'th and first momentum of the density distribution function as follows:

$$
\begin{aligned}
& \rho=\sum_{\alpha} f_{\alpha} \\
& \rho \vec{V}=\sum_{\alpha} \vec{e}_{\alpha} f_{\alpha}
\end{aligned}
$$

In the assumption of low Mach number $\left(M a=\|\vec{V}\| / c_{s}<<1\right)$ invoked as the nearly incompressible limit is approached, the macroscopic continuity and Navier-Stokes equations can be recovered from the evolution equation of the density distribution function through the Chapman-Enskog expansion. The final forms of the continuity and Navier-Stokes equation are: 


$$
\begin{aligned}
& \nabla .(\vec{V})=O\left(\delta^{2}\right) \\
& \partial_{t} \vec{V}+\vec{V} \cdot \nabla \vec{V}=-\frac{\nabla p}{\rho}+v \nabla^{2} \vec{V}+O\left(\delta^{2}\right)
\end{aligned}
$$

where $\delta$ is a small parameter proportion to Knudsen number (Hou et al., 1995), $p=\rho c_{s}^{2}$ is the pressure from the equation of the state for the ideal gas, $c_{s}=c / \sqrt{3}$ is the sound speed and the kinetic viscosity is given by (He et al., 1998):

$$
v=\frac{\left(2 \tau_{v}-1\right)}{6} \frac{(\delta x)^{2}}{\delta t}
$$

The new 2D thermal model proposed by (Peng et al., 2003), is based on the idea of omitting the viscous heat dissipation and compression work done by pressure, while this term remains in the thermal model formulated by (He et al., 1998). Moreover, the energy equation can be recovered through the Chapman-Enskog expansion in second order of accuracy, more details for the derivation process can be found in (Peng et al., 2003).

The evolution equation for the Peng's energy distribution model is:

$$
g_{\alpha}\left(\vec{x}+\vec{e}_{\alpha} \delta t, t+\delta t\right)-g_{\alpha}(\vec{x}, t)=-\frac{1}{\tau_{c}}\left(g_{\alpha}(\vec{x}, t)-g_{\alpha}^{e q}(\vec{x}, t)\right)
$$

The correspondent equilibrium energy distribution function following the work of (He et al., 1998) is:

$$
g_{\alpha}^{e q}=\omega_{\alpha} \rho \varepsilon\left[\frac{3\left(\vec{e}_{\alpha}^{2}-\vec{V}^{2}\right)}{2 c^{2}}+3\left(\frac{3 \vec{e}_{\alpha}^{2}}{2 c^{2}}-1\right) \frac{\left(\vec{e}_{\alpha} \cdot \vec{V}\right)}{c^{2}}+\frac{9}{2} \frac{\left(\vec{e}_{\alpha} \cdot \vec{V}\right)^{2}}{c^{4}}\right]
$$

where $\varepsilon=D R T / 2, \mathrm{R}$ is the gas constant and $\mathrm{D}$ is the spatial dimension. Then the macroscopic temperature is computed simply by:

$$
\rho \varepsilon=\sum_{\alpha} g_{\alpha}
$$

As for the continuity and Navier-Stokes equation and following the works of (Hou et al., 1995) the Chapman-Enskog expansion for the new thermal energy distribution function can recover the macroscopic energy equation, the final result is as below:

$$
\partial_{t}(\rho \varepsilon)+\nabla \cdot(\rho \vec{V} \varepsilon)=\chi \nabla^{2}(\rho \varepsilon)+O\left(M^{2} \delta T\right)
$$


and the diffusivity $\chi$ is determined by:

$$
\chi=\frac{1}{3}\left(2 \tau_{c}-1\right) \frac{(\delta x)^{2}}{\delta t}
$$

\subsection{Buoyancy force and dimensionless parameters}

For natural convection flows, the additional body force term, $F_{\alpha}$, can be formulated by the Boussinesq approximation. The Boussinesq approximation considers that all the properties of the fluid are constant, except the fluid density given by $\rho=\rho_{r}\left(1-\beta\left(T-T_{r}\right)\right)$, where $\rho_{r}$ is a reference fluid density, $\beta$ is the thermal coefficient expansion of the fluid and $T_{r}$ is a reference fluid temperature, then the external buoyant force $\rho_{r} \vec{G}=-\rho_{r} \beta\left(T-T_{r}\right) \vec{g}$ appearing in Navier-Stokes equations will be expressed in equation [1] as:

$$
F_{\alpha}=\frac{\vec{G} \cdot\left(\vec{c}_{\alpha}-\vec{V}\right)}{c_{s}^{2}} f_{\alpha}^{e q}
$$

Following these considerations $\|\vec{V}\|<<c_{\alpha}, f_{\alpha}^{e q} \approx w_{\alpha} \rho(x, t)$, and $T_{r}=0$, the final form of the external body force is:

$$
F_{\alpha}=3 w_{\alpha} \cdot \rho(\vec{x}, t) \cdot g_{y} \cdot \beta T(\vec{x}, t) \cdot c_{\alpha y}
$$

To guarantee the incompressible limit, we choose a characteristic velocity flow $U=(g \beta \Delta T H)^{0.5}$, where $\Delta T=1$ and $H$ denotes the number of nodes in the vertical direction. The Prandtl number and Rayleigh number are defined as:

$$
\operatorname{Pr}=\frac{v}{\chi}, R a=\frac{g \beta \Delta T H^{3}}{v \chi}
$$

thus, the kinetic viscosity $v$ is related to $H$ (number of nodes) as:

$$
H=\frac{\sqrt{3}}{M a} \sqrt{\frac{R a}{P r}} \cdot v
$$

Means that after choosing the appropriate Mach number value and for a given Rayleigh number, it is required a minimum value of the viscosity $v$ to ovoid instabilities as mentioned in (Miller, 1994).

To investigate the enhancement of the thermal transfer, the average Nusselt number, $\overline{N u}$ and the left Nusselt number $N u_{0}$, are estimated using the following formula: 


$$
\overline{N u}=1+\frac{\langle u T\rangle}{\chi \cdot \Delta T / W}
$$

\langle\rangle denotes the average value on the whole domain.

\subsection{Implementation of the boundary conditions}

The bounce-back rule of the non-equilibrium distribution function proposed by (Zou et al., 1997) is used for the boundary condition. At the hot wall (left), for instance, the outgoing distribution functions (from the flow to the wall) $f_{2}, f_{3}, f_{4}, f_{6}$ and $f_{7}$ are determined by the streaming process and the incoming ones (from the wall to the flow) $f_{1}, f_{5}$ and $f_{8}$ obey to the bounce-back treatment. These density distribution functions at the boundary should satisfy the following condition:

$$
f_{\alpha}^{n e q}=f_{\beta}^{n e q}
$$

where $\vec{e}_{\alpha}$ and $\vec{e}_{\beta}$ have opposite directions. The energy distribution function at the boundary satisfies:

$$
g_{\alpha}^{\text {neq }}-\vec{e}_{\alpha}^{2} f_{\alpha}^{\text {neq }}=-\left(g_{\beta}^{\text {neq }}-\vec{e}_{\beta}^{2} f_{\beta}^{\text {neq }}\right)
$$

The wall temperatures are used for the calculation of the internal energy equilibrium functions.

The Dirichlet boundary condition at the stationary walls is transformed to Neumann boundary condition using the popular second order accurate relation:

$$
T_{0, j}=\left(4 T_{1, j}-T_{2, j}\right) / 3
$$

\section{Simulation the differentially heated square cavity}

\subsection{Convergence criteria and convergence rate}

In the simulations, the $2 \mathrm{D}$ was mapped using a square lattice, where $\delta x=\delta y$ ( $\delta x=\delta y=\Delta x=\Delta y$ ) for the $\mathrm{D} 2 \mathrm{Q} 9$ model. The aspect ratio $A r=W / H$ was equal to the unit. The Prandtl number was assumed to be constant with a value 0.71 and the Rayleigh number varies from $10^{3}$ to $10^{6}$. 
In our calculations, we opt for the coarser mesh sizes that allow a deviation around or smaller than $1 \%$ when the steady state is reached, it is found to be $48^{2}$, $80^{2}, 128^{2}$ and $176^{2}$ for respectively $10^{3}, 10^{4}, 10^{5}$ and $10^{6}$.

To ensure the computation convergence we opt, two criteria is defined as follows

$$
\left|\frac{\overline{N u}(t)-\overline{N u}(t+5000)}{\overline{N u}(t)}\right| \leq 10^{-6}
$$

means that $\overline{\mathrm{Nu}}$ changes less than $10^{-6}$ after 5000 time-step

In addition, (Paolucci et al., 1989) monitored the oscillations in $u, v$ and $T$ at the location $(x / H, y / H)=(0.1032,0.8036)$. To show, also, the achievement of steady state we will use the same monitoring point.

The grid independency is checked for $\mathrm{Ra}=10^{4}$ using several grid sizes varying from $32^{2}$ to $224^{2}$. In this test case, we compute the relative error in $\overline{N u}$ and we suppose the next relationship between the relative error $E$ and the lattice step $\Delta x$.

$$
E=C \cdot(\Delta x)^{a}
$$

Here $C$ is a constant, $a$ is the order of accuracy of the model, $\Delta x=1 / n$ and $n$ is the number of nodes for each direction of the square cavity.

The results of $\ln (E)$ versus $\ln (\Delta x)$ are plotted in Figure 3, the equation of the obtained fitting curve shows that $a \approx 1.95$. Then, as first result of this part, it is clear that the Nusselt number solution converge at the rate of second order. The slightly distortion via $a=2$ (the order of accuracy of LBM for recovering N-S equations) may be attributed to the bounce-back scheme used at the boundaries (first order accurate), the truncation error in the equilibrium distribution function and the round-off error (in formula [15] for example).

In this study we have seen that when the number of nodes in each direction increases, the calculated value of $\overline{N u}$ approaches the benchmark result, and further more, when the number of nodes increases from 176 to 224, there is not much effect on the result. Hence, the grid independency is a second view of this part. 


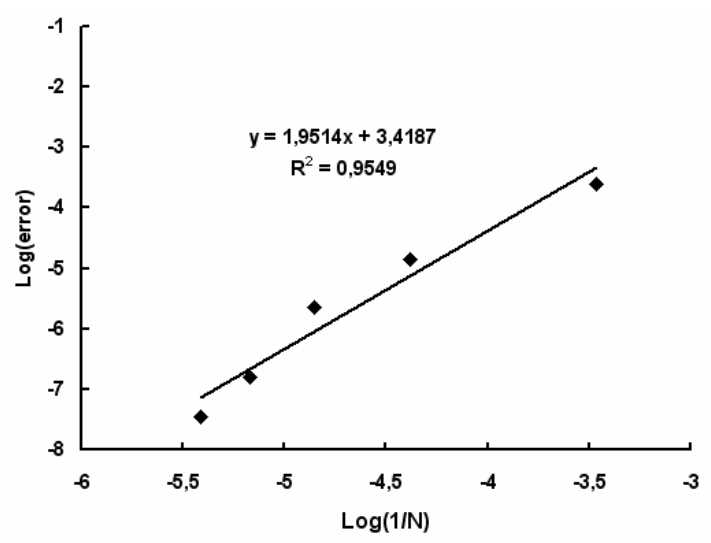

Figure 3. Numerical error versus lattice spacing for square cavity

\subsection{Laminar flow}

Table 1 shows the numerical solutions of the maximum horizontal velocity at the mid-height and the maximum vertical velocity at the mid-width $\left(u_{\max }\right.$ and $v_{\text {max }}$ respectively) normalized by the reference velocity $\chi / H$, the maximum stream function magnitude and the average Nusselt number value $\overline{\mathrm{Nu}}$ of the whole of the cavity.

Through the Table 1 we can say that the present results are in excellent agreement with those obtained by (de Vahl Davis, 1983). The maximum relative deviations are $1.069 \%$ and $0.988 \%$ obtained for $v_{\text {max }}$ and $\overline{N u}$ (resp.) at $\mathrm{Ra}=10^{6}$, and elsewhere the deviation is smaller than $0.767 \%$.

Furthermore we can observe that the Nusselt number obtained by this model is slightly smaller than the Navier-Stokes result, this is certainly due to the truncated equilibrium distribution function by the so-called low-Mach number approximation $\left(O(M a)^{2}\right)$.

Figure 4 shows the contour maps of the streamlines (a), which shows the flow pattern, and the temperature field (b) for $R a=10^{3}, 10^{4}, 10^{5}$ and $10^{6}$.

These plots agree well with results obtained by (Shu et al., 1998), in addition it is visibly that the property of symmetry which was well studied by (de Gassowski et al., 2003) is highly shown through the layouts of the streamlines and of temperatures, this proves that the LBM is a tool able to represent the convection problems with high order of accuracy.

In the other side, it is plotted in Figure 5 the horizontal velocity $u$ vs $y$ at mid-width (a) and the vertical velocity $v$ vs $x$ at mid-height (b) for the four Rayleigh numbers. At first, we can say that the property of symmetry is also present for the velocity fields. In other side, it is clear that when the Rayleigh number increases the location of 
$\left|v_{\text {max }}\right|$ moves to the wall, this agrees with the stretched streamlines near the heated and cooled walls Figure 4 . However, the location of $\left|u_{\max }\right|$ moves to the top and bottom walls until $\mathrm{Ra}=10^{5}$ but bounces for $\mathrm{Ra}=10^{6}$, this will be discussed below.

Table 1. Comparison of the present results at different Rayleigh numbers with results of Ref. [a] ${ }^{1}$

\begin{tabular}{|c|c|c|c|c|c|}
\hline & $\mathrm{Ra}$ & $10^{3}$ & $10^{4}$ & $10^{5}$ & $10^{6}$ \\
\hline \multirow{2}{*}{$u_{\max }$} & Present & 3.634 & 16.134 & 34.662 & 64.511 \\
\hline & Ref. [a] & 3.649 & 16.190 & 34.736 & 64.775 \\
\hline \multirow{2}{*}{$v_{\max }$} & Present & 3.674 & 19.526 & 68.216 & 218.281 \\
\hline & Ref. [a] & 3.698 & 19.638 & 68.640 & 220.640 \\
\hline \multirow{2}{*}{$\left|\psi_{\text {max }}\right|$} & Present & 1.165 & 5.053 & 9.614 & 16.767 \\
\hline & Ref. [a] & $1.174^{2}$ & $5.071^{1}$ & 9.612 & 16.750 \\
\hline \multirow{2}{*}{$\overline{N u}$} & Present & 1.115 & 2.226 & 4.508 & 8.713 \\
\hline & Ref. [a] & 1.118 & 2.243 & 4.519 & 8.800 \\
\hline
\end{tabular}
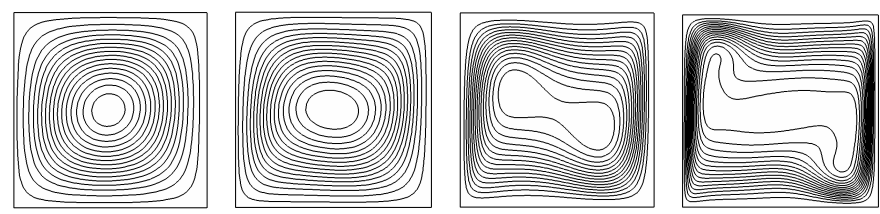

(a)
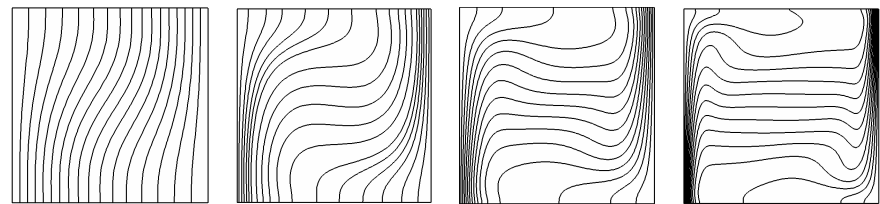

(b)

Figure 4. Contour maps of streamlines (a) and temperature field (b) at (from left to right) $R a=10^{3}, 10^{4}, 10^{5}$ and $10^{6}$

1. de Vahl Davis (1983)

2 . value corresponding to $\left|\psi_{\text {mid }}\right|$ 


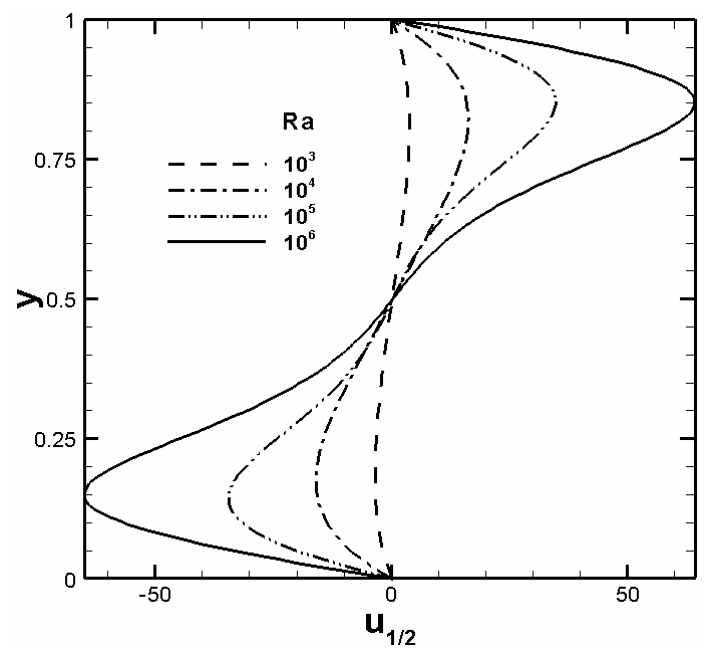

(a)

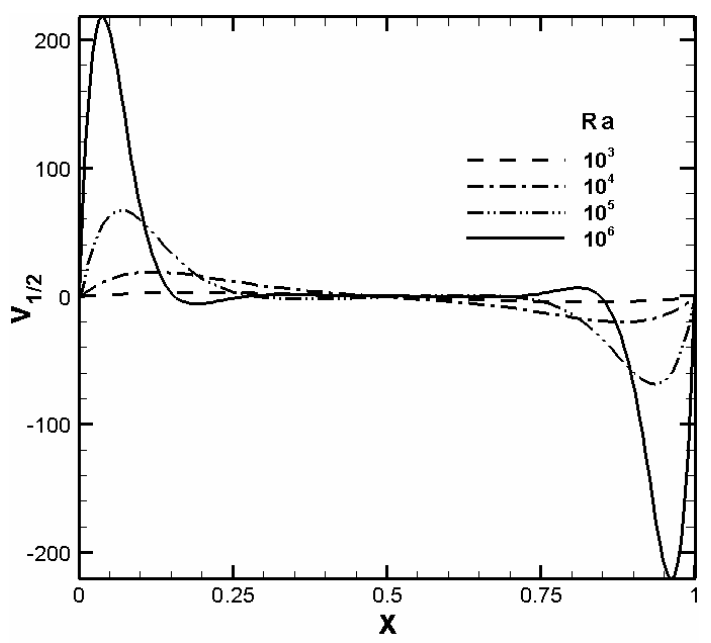

(b)

Figure 5. Plots of $u_{1 / 2}(y)$ (a) and $v_{1 / 2}(x)(b)$ for $R a=10^{3}, 10^{4}, 10^{5}$ and $10^{6}$

It is worthy to explain some working phenomena and behaviors that caused by increasing the Rayleigh number. For the Rayleigh number range from $10^{3}$ to $10^{4}$, a circular cellular structure dominates the stream-function field as illustrated in Figure 4(a). For higher values of the Rayleigh number, one can observe a temperature undershoot and a stationary wave-like structure form, at the up-left and low-right corners where the flow discharges from the thin vertical boundary layer into the core flow, this is due to the fact that high Rayleigh number enhances the fluid motion. In 
addition, the thermal and dynamic boundary layer thicknesses decrease with icreasing Rayleigh number. This process of shooting is clearly remarked for $\mathrm{Ra}=10^{6}$. Indeed, the upper-left corner of the streamlines is filled by a stretched lines that will be, later, rejected far from the middle of the upper wall, and this is the reason of behind the bounce of the location of $\left|u_{\text {max }}\right|$ cited above. One can also see the establishing lamellate thermal structure when increasing the Rayleigh number, thus we say that the flow presents a stratified structure. The overshoot and undershoot of the flow patterns between the vertical boundary layers and the core region become more pronounced when $R a$ increases and will induce a large core region and a divergence of the horizontal wall boundary layers. The main flow is moving along the hot and cold wall and leaves the adiabatic side walls before it reaches the isotherm walls.

Through these funding, one can say that the LB results have a very good agreement with those of other methods in both qualitative and quantitative senses at little for the laminar flow.

\subsection{Relationship between heat transfer and convection intensity}

Formers numerical and experimental works in square cavity showed that the Nusselt number and the Rayleigh number are related by the power law $\overline{N u} \propto(R a)^{b}$ in which " $b$ " is generally close to 0.3 . In the present study, the plot of the best-fitted curve of Nusselt numbers $\overline{N u}$ versus the Rayleigh number in the range $10^{3} \leq R a \leq 10^{6}$ is found to lie along straight line when plotted as $\log (\overline{N u})$ versus $\log (\mathrm{Ra})$ and then, the power relationship $\overline{N u}=0.1429 R a^{0.2982}$ is obtained. The present obtained correlation are in good agreement with these established by Berkovsky and Polevikov in (Baïri et al., 2007) which is rectified by Catton for laminar natural convection in rectangular cavities. In our computations corresponding to $A r=1$, we have observed that $\overline{N u} \approx N u_{0}$, where $N u_{0}$ is left Nusselt number and also $N u_{0}=0.1478 R a^{0.2965}$. This result can be explained by the fact that, in steady state, the heat flux given at the hot wall is transferred within the domain and is received at the cold wall. Moreover, the average Nusselt number is slightly little then the left one because of the adiabatic walls effects, the average Nusselt number profile changes (a decrease) along x-direction near insulated walls. In other side the exponent of the power law is generally taken $1 / 3$, in this case we obtain $N u_{0}=0.0906 R a^{1 / 3}$, this new power law agrees well with the experimental result of (Ozoe et al., 1998) where $N u=0.109 R a^{1 / 3}$, then a small deviation $0.43 \%$ in $N u_{0}$ for $R a=10^{6}$.

\section{Effects other secondary parameters}

Several parameters can influence the dynamic and thermal behavior in a differentially heated cavity. We study in this part the effect of the geometry of the cavity, of its configuration and the effect of the nature of the fluid modeled respectively by the aspect ratio $A r$, the angle of inclination of the cavity $\gamma$ and the Prandtl number 
Pr. In this section, first we study the effects of these parameters separately, on the left Nusselt number, and then we treat the coupled effects. The Rayleigh number is chosen to be $10^{3}, 10^{4}$ and $10^{5}$ for all the simulations for the fact that the convection mode is preponderant and to be far from instabilities phenomena.

\subsection{Effect of the aspect ratio Ar}

The effect of the aspect ratio on flow and heat in air-filled cavity has been mentioned in full with former works, this geometry find its application in melting phenomena. When the solid part starts to melt, the aspect ratio $W / H$ increases and one has a tall/slender cavity, on the contrary, one will have a shallow cavity. The change of geometry induces the change of critical thresholds, such as the critical Rayleigh, $R a_{c}$, of transitions to time-dependence and chaotic flow.

These transitions occur at broadly different values of $R a$, indicating a strong influence of the aspect ratio on which various works are undertaken.

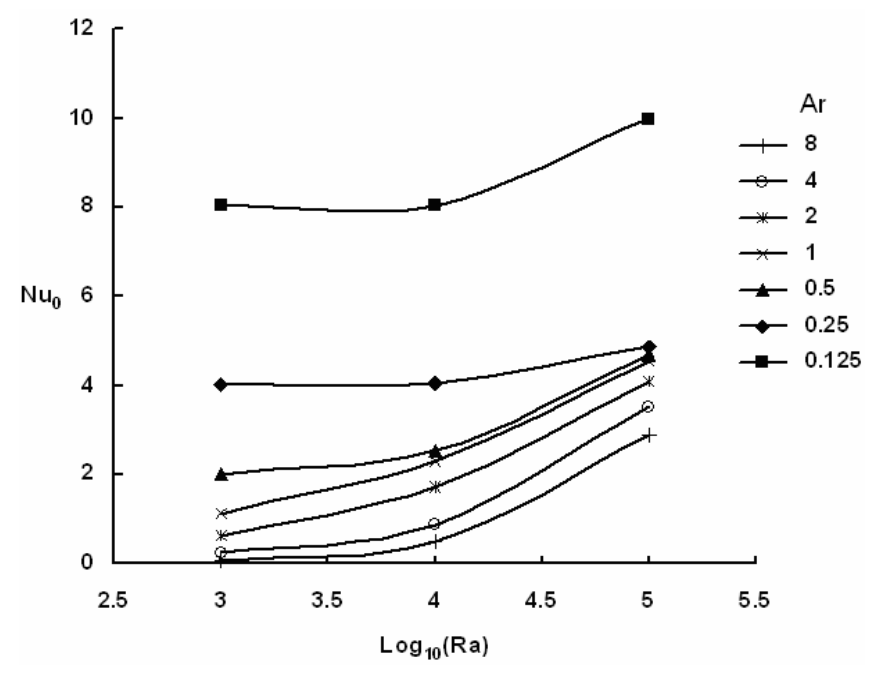

Figure 6. Aspect ratio effect on heat transfer for $10^{3} \leq R a \leq 10^{5}$

For $R a=10^{3}$ and $A r=0.5$ the plot of the temperature field shows vertical lines, then the heat transfer is purely conductive. This can be concluded also from the value of average Nusselt number $\overline{N u}$ which is close to 1 , but $N u_{0}=2.026$ and increases too with the decrease of $A r$. On the contrary when $A r$ increases $(>1) N u_{0}$ decreases and $\mathrm{Nu}$ increases excessively, the heat transfer is obviously purely convective. These remarks are in conformity with the results obtained by (K.A.R. Ismail et al., 2000). The plots of $N u_{0}$ versus $R a$ for each aspect ratio are shown in Figure 6. 
The major idea of this subsection is to show that there is an aspect ratio $(\approx 0.25)$ where the Nusselt number is almost independent from the Rayleigh number in this range. Likewise the dependence is strict.

\subsection{Effect of the used fluid}

The Prandtl number formula shows a balance of "volatility" between the velocity and the temperature field. Then, the nature of the fluid can also affect the heat and fluid flow. Figure 7 presents the evolution of the Nusselt number versus the Prandtl number in the range $0.025 \leq P r \leq 6$ for different values of Rayleigh number.

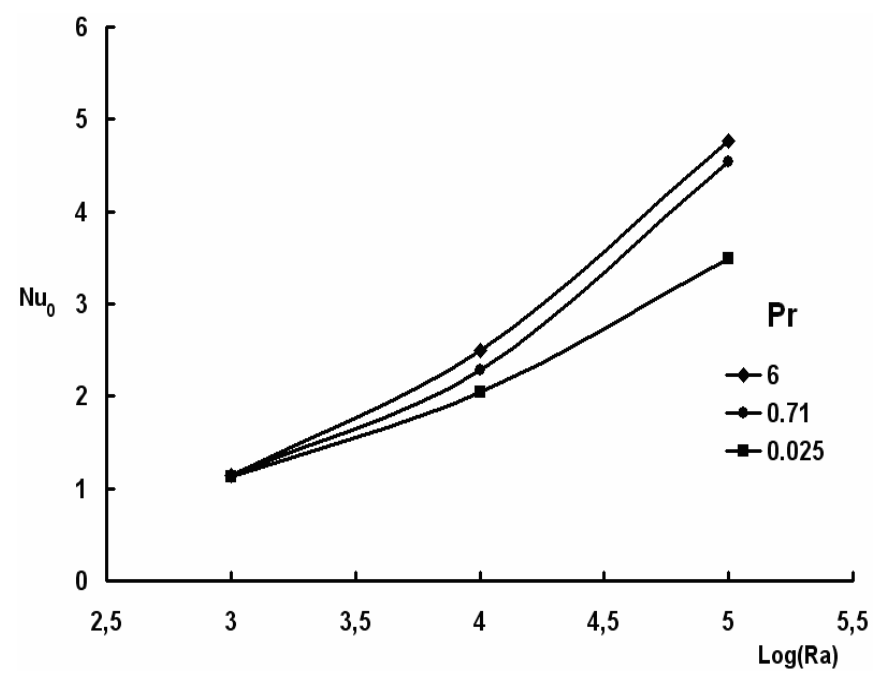

Figure 7. Prandtl number effect on heat transfer at various Rayleigh numbers

One can see that heat transfer increases with increasing Prandtl number from 0.025 (corresponding to the liquid metal, such as gallium and mercury) to 6 (characteristic of water). A fixed Rayleigh number, the heat transfer pattern remains relatively unchanged for moderately large values of the $\operatorname{Pr}$ (up to 1). This goes well with the question of volatility: for high Prandtl number the $(\chi \prec \prec v)$ the temperature is less volatile then the velocity.

\subsection{Effect of the inclination}

The present part provides numerical simulations of natural convection flow in a wide variety of orientations. Only the momentum equation will be affected by the change of the angle of inclination at Boussinesq term. The configuration of an in- 
clined cavity is presented in Figure 8. The Rayleigh number is chosen to be $10^{5}$. The results show agreements with previous work in quantitative sense.

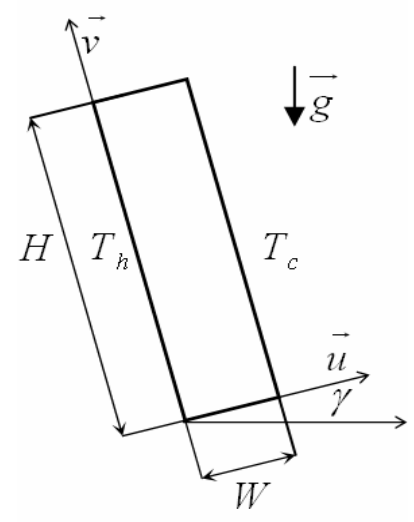

Figure 8. Configuration of the inclined heated cavity

The results for $A r=1$ are plotted in Figure 9 and are found to reproduce the same profile as that obtained by (Ozoe et al., 1975). One can observe that heat transfer increases when the angle $\gamma$ increases to a value close to $15^{\circ}$, the correspondent Nusselt number is 4.7. In such configuration and for the earlier inclinations, the ybuoyancy component decreases $(\cos (\gamma))$ and the $\mathrm{x}$-buoyancy component increases $(\sin (\gamma))$; thus, it enforces the convective motion between the isothermal walls. With further increasing of $\gamma$, the value of $\overline{N u}$ decreases considerably to achieve a minimum at a critical angle between $90^{\circ}$ and $100^{\circ}$. The correspondent Nusselt number of Rayleigh-Bénard configuration $\left(90^{\circ}\right)$ is 3.85. When $\gamma$ increases more, $\overline{\mathrm{Nu}}$ increases to exceed the value obtained at $\gamma=0^{\circ}$ and certainly to reach again the value 4.7 at $\gamma=165^{\circ}$. Once more, $\gamma$ increases but $\overline{N u}$ decreases clearly to be the unit in a configuration of enclosure heated from above $\left(\gamma=270^{\circ}\right)$.

This value implies pure diffusive heat transfer. In this configuration, the density of the particle heated at the top of the cavity decreases, but the tendency of the particles is to ascend again, thus the agitation will be located at the top, so the whole convective heat decreases.

In order to show the flow structure, a number of configurations are presented in Figure 10. The inclination angles are $0^{\circ}, 45^{\circ}, 90^{\circ}, 225^{\circ}$ and $270^{\circ}$. We note here that in Figure 10 , from $0^{\circ}$ to $270^{\circ}$, a step of $45^{\circ}$ is chosen, but the plots corresponding to $135^{\circ}$ and $180^{\circ}$ will not be sown for symmetry raison. The case of $\gamma=0^{\circ}$, the thermal structure expresses a stratification tendency. Increasing $\gamma$ to $45^{\circ}$, we observe that the thermal transfer is concentrated near the adiabatic walls; however a flatten structure is obtained for the dynamic structure. For the Rayleigh-Bénard case, a rotating motion dominates the core of the thermal structure and the transfer is more pushed 
to the walls, which decreases the convective transfer between the isothermal walls. The dynamic pattern is a concentric circles with two small vortices at the coins.

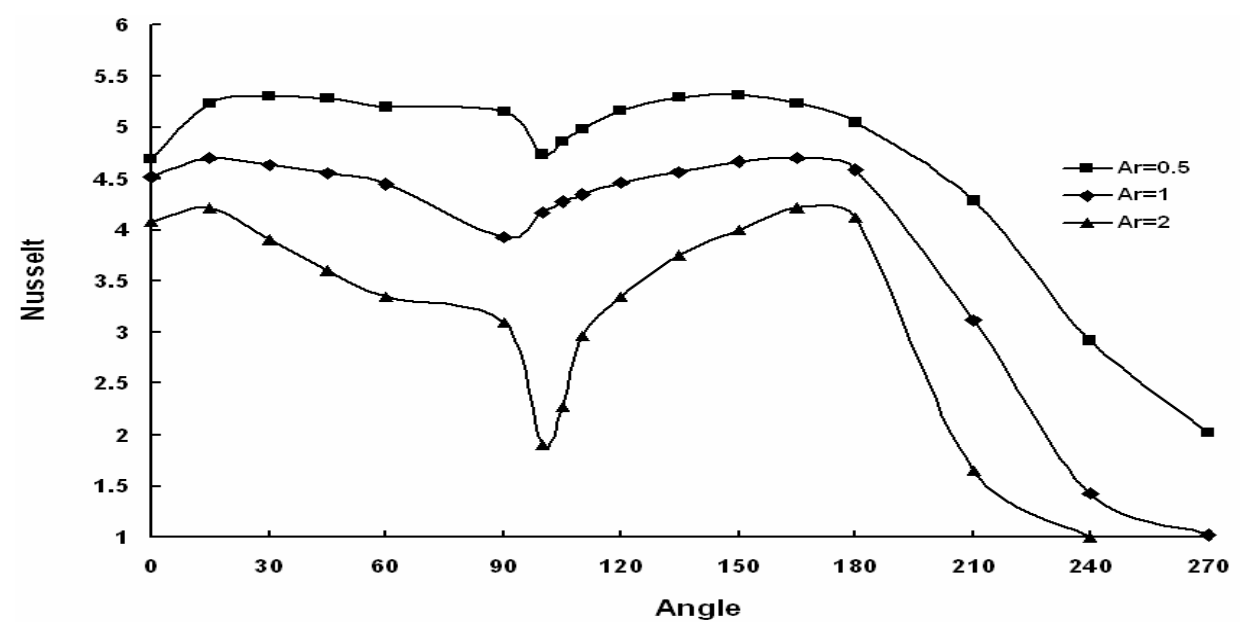

Figure 9. Coupled effects of aspect ratio and inclination on the Nusselt number for $\operatorname{Pr}=0.71$

Increasing $\gamma$ to $225^{\circ}$, the conductive effects begin and no stretched thermal boundary layers, and moreover at $\gamma=270^{\circ}$ which corresponds to a cavity heated from top, so, a purely conductive regime take place. In this case, the isothermal lines become strict horizontal lines. The heat transfer is the least.

\subsection{The coupled effects}

Modeling phenomena is a engineering tool. For such technique previous researches using standard Navier-stokes solvers have been interested to establish correlations for Nusselt number as a function of the different parameters cited above (Robert, 1999). In this part we are interested to study the coupled effects of the inclination and the aspect ratio at the same time on heat transfer without establishing correlation. Two aspect ratios 0.5 and 2 are investigated. The results obtained are reported in Figure 9.

For $A r=0.5$ no change observed in the thermal behavior of flow, the same profile is obtained but quantitatively the Nusselt number increases and the profile is translated due to the conductive effect. However, for $A r=2$ the flow becomes periodic at $\gamma=90^{\circ}$. The dynamic flow structure changes many times at one period and the dimensionless frequency is close to 15.86 (in unit of $H^{2} / \chi$ ). The correspondent timeaveraged Nusselt number at the isotherm wall is close to 3.16. Figure 11 shows the Nusselt number time-historic when the regime is established. The flow is also periodic 
for $\gamma=100^{\circ}$, the dimensionless frequency is 21.93 and the time-averaged Nusselt number is 2.06; The streamlines show a small cell near the hot wall and a great cell near the cold wall which is three times the small one. At $\gamma=105^{\circ}$ the flow is found to be chaotic; however, for $\gamma=110^{\circ}$ the flow becomes steady and it remains steady for $\gamma \geq 110^{\circ}$. The result will certainly change if the Rayleigh number values change.
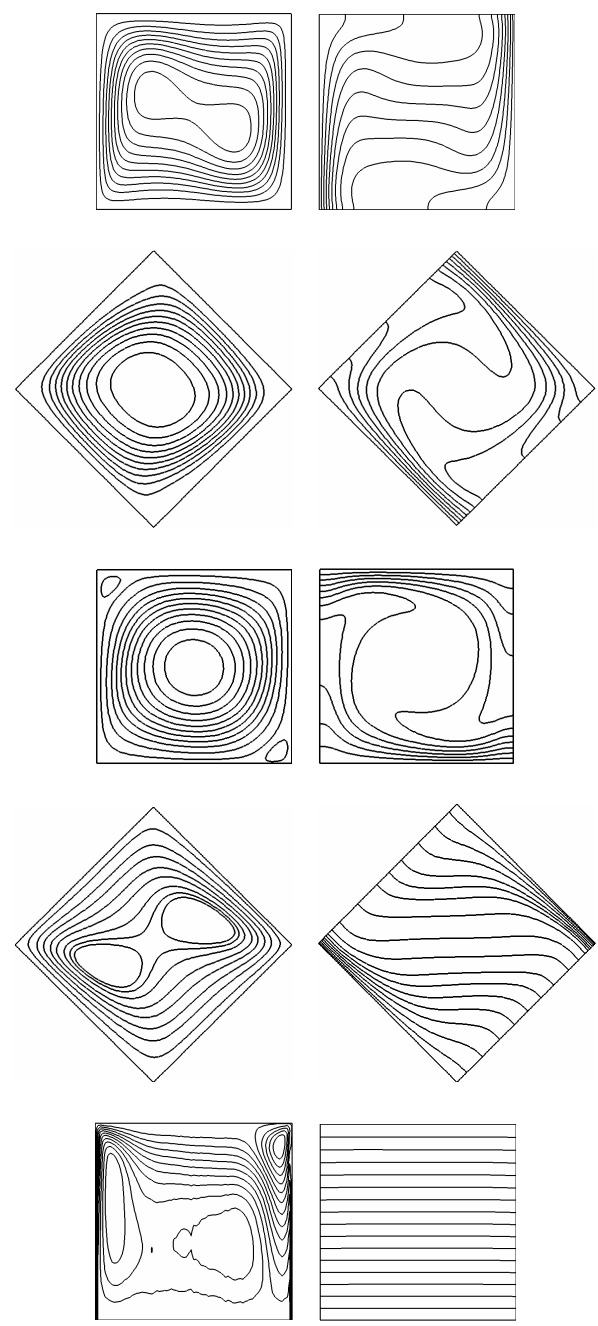

Figure 10. Inclination effects on dynamic and thermal behaviors, stream function (left) and isotherms (right) for $\gamma=0^{\circ}, 45^{\circ}, 90^{\circ}, 225^{\circ}$ and $270^{\circ}$ 


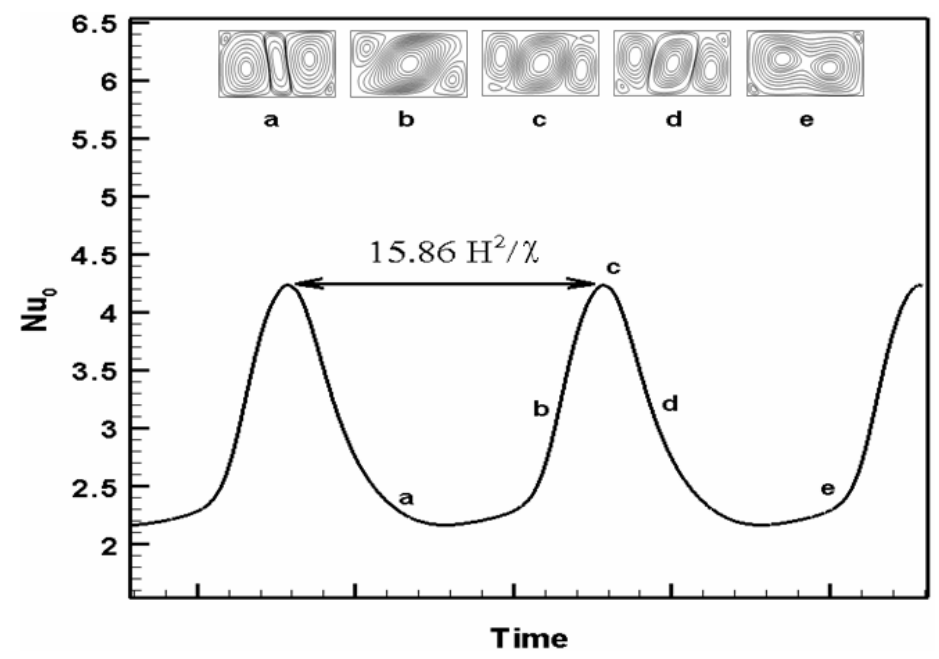

Figure 11. Dynamic behaviour under coupled effect of aspect-ratio and inclination

The effect of the angle of inclination comes to be perceptible with the increase of the aspect ratio. As it is shown in Figure 9 heat transfer depends strongly on the aspect ratio. The Nusselt number decreases with the aspect ratio. In the other side the coupled effect coming from the increase of the aspect ratio(upper to unit) and the increase of the angle of inclination (upper to $90^{\circ}$ ) is expressed by the displacement of angle at minimum and maximum transfer (Nusselt number) towards the limit $180^{\circ}$. As a better result of this part is to show that for $A r=0.5$ (or slightly different) the heat transfer is quasi-independent from the angle of inclination from 15 to $90^{\circ}$.

For low aspects ratio the isotherm walls become approached $(H=1, W<1)$, the heat transfer is in the majority conductive and will be less influenced by changing the inclination even for $\gamma$ near $90^{\circ}$, the Reyleigh-Bénard rolls establish and move the flow. However, for high aspects ratio $(H=1, W>1)$, the heat transfer is in the majority convective (driven by velocity). The flow regime changes to periodic at $\gamma=90^{\circ}$ and $\gamma=100^{\circ}$, it is chaotic at $\gamma=105^{\circ}$, and then it is more influenced by changing the inclination.

\section{Conclusion}

The LBM is used to simulate an incompressible natural convection flow of Boussinesq fluid within a differentially heated rectangular cavity. The LBM is found to be an effective and convenient alternative to simulate laminar flow in confined space. 
To show the accuracy of this LB model, we have conducted a comparative study on heat and fluid flow in heated square cavity. The chosen grid sizes yields to the same order of accuracy as the other solvers and shows that high monitoring numbers needs high grid resolution to get accurate solutions.

The effects of the secondary parameters (geometry, configuration and fluid properties) on the heat and fluid flows have been carried out. The results of the LBM are found to be in good agreement with former works. It has been found that dynamic and thermal behaviour in heated differentially rectangular cavity depends greatly on aspect ratio, low Prandtl numbers and inclination but marginally on high Prandtl numbers. The coupled effects of these parameters can change completely the regime of the flow.

In our study we are limited to low Rayleigh numbers. High Rayleigh numbers are characterized by thin boundary layers, so a grid refinement or more nodes are needed. In other side, the common problem in simulating fluid flows is the modeling of turbulence (more details in (Succi, 2001; Huidan Yu, 2005) and (Shu, 2006)). An efficient tool for handling arbitrary meshes and turbulent state and to be combined with various models of turbulence is TLLBM, a meshless efficient tool recently developed by C. Shu. The next step in our works is to introduce, first, a grid refinement using the TLLBM approach and, second, a large eddy simulation modeling of turbulence.

\section{References}

Bouzidi M., D’Humières D., Lallemand P., Luo L.S., "Lattice Boltzmann equation on a twodimensional rectangular grid", J. Comput. Phys., 172, 2001, p. 704-717.

Bouzidi M., Firdaouss M. and Lallemand P., "Momentum transfer of a Boltzmann-lattice fluid with boundaries", Phys. Fluid, 13, 2001, p. 3452-3459.

Chen S. and Doolen G. D., "Lattice Boltzmann method for fluid flows", Annu. Rev. Fluid Mech., 30, 1998, p. 329-64.

de Gassowski G., Xin S. et Daube O., «Bifurcations et solutions multiples en cavité 3D différentiellement chauffée », C. R. Mécanique, 331, 2003, p. 705-711.

de Vahl Davis G., "Natural convection of air in a square cavity: A benchmark numerical solutions", Int. J. Numer. Methods Fluids, 3, 1983, p. 249-264.

D'Humières D., Bouzidi M., Lallemand P., "Thirteen-velocity three-dimensional lattice Boltzmann model", Phys. Rev. E, 63, 2001, 066702.

Dubois M., Une introduction aux gaz de Boltzmann sur réseau, CNAM, Paris, 2006.

Filippova O. and Hanel D., "Acceleration of Lattice-BGK schemes with grid refinement", Journal of Computational Physics, 165, 2000, p. 407-427.

Guo Z. and Zhao T. S., “A Lattice Boltzmann model for convection heat transfer in porous media", Numerical Heat Transfer, Part B, 47, 2005, p. 157-177. 
Guo Z. and Zhao T. S., "Explicit finite-difference lattice Boltzmann method for curvilinear coordinates", Physical Review, E 67, 066709, 2003.

Guo Z., Shi B. and Zheng C., "A coupled lattice BGK model for the Boussinesq equations", Int. J. Numer. Meth. Fluids, 39, 2002, p. 325-342.

He X. and Luo L. S., "Lattice Boltzmann model for the incompressible Navier-Stokes equation”, Journal of Statistical Physics, vol. 88, n³/4, 1997.

He X., Zou Q., Luo L. S. and Dembo M., “Analytic Solutions of Simple Flows and Analysis of Nonslip Boundary Conditions for the Lattice Boltzmann BGK Model", Journal of Statistical Physics, vol. 87, $\mathrm{n}^{\circ} 1 / 2,1997$.

Hou S. and Zou Q., "Simulation of cavity flow by the Lattice Boltzmann method", Journal of Computational Physics, 118, 1995, p. 329-347.

Ismail K. A. R., Scalon V. L., "A finite element free convection model for the side wall heated cavity", Int. J. Heat Mass Transfer 43, 2000, p. 1373-1389.

Jami M., Mezrhab A., Bouzidi M., Lallemand P., "Lattice Boltzmann method applied to the laminar natural convection in an enclosure with a heat-generating cylinder conducting body", International Journal of Thermal Sciences, 46, 2007, p. 38-47.

Kerr R. M. and Herring J. R., "Prandtl number dependence of Nusselt number in DNS", J. Fluids Mech, 1999.

Lallemand P., Luo L. S., "Lattice Boltzmann method for moving boundaries", Journal of Computational Physics, 184, 2003, p. 406-421.

Mezrhab A., Jami M., Bouzidi M. and Lallemand P., "Analysis of radiation-natural convection in a divided enclosure using the lattice Boltzmann method", Computers and fluids, $36, \mathrm{n}^{\circ} 2,2007$, p. $423-434$.

Mohamad A. A., Applied Lattice Boltzmann Method for Transport Phenomena, Momentum, Heat and Mass Transfer, 2007.

Ozoe H., Sayama H., "Natural convection in an inclined rectangular channel at various aspect ratios and angles-Experimental measurements", Int. J. Heat Mass transfer, vol. 18, 1975, p. $1425-1431$.

Paolucci S. and Chennoweth D. R., "Transition to chaos in a differentially heated vertical cavity", J. Fluid Mech., vol. 201, 1989, p. 379-410.

Peng Y., Shu C., Chew Y. T., "Simplified thermal lattice Boltzmann model for incompressible thermal flows", Phys. Rev., E, 68, 026701, 2003, p. 1-8.

Qian Y. H., D’Humieres D. and Lallemand P., "Lattice BGK Models for Navier-Stokes Equation”, Europhys. Lett., vol. 17, n 6, 1992, p. 479-484.

Succi S., The lattice Boltzmann equation for fluid dynamics and beyond, Italy, 2001, p. 15

Semma E., El Ganaoui M., Bennacer R., Mohamad A. A., "Investigation of flows in solidification by using the lattice Boltzmann method", International Journal of Thermal Sciences, 47, 2008, p. 201-208. 
Shu C. and Xue h., "Comparison of two approaches for implementing stream function boundary condition in DQ simulation of natural convection in square cavity", Int. J. Heat and Fluid Flow, 19, 1998, p. 59-68.

Shu C., Niu X.D., Peng Y. and Chew Y.T., "Taylor series expansion-and least square-based Lattice Boltzmann method: an efficient approach for simulation of incompressible viscous flows", Progress in Computational Fluid Dynamics, vol. 5, 2005, Nos. 1/2.

Shu C., Peng Y., and Chew Y. T., "Simulation of natural convection in square cavity by Taylor series expansion-and least square-based lattice Boltzmann method", International Journal of Modern Physics C, vol. 13, n 10, 2002, p. 1399-1414.

Sukop M. C., Thorne Daniel T., Lattice Boltzmann modelling, an introduction for Geoscientists and engineers, Florida USA, 2005

Vahala G., Pavlo P., Vahala L. and Martys N. S., "Thermal Lattice Boltzmann Model (TLBM) for compressible flows", International Journal for Modern Physics C, vol. 9, 1998, n $^{\circ} 8$, p. $1247-1261$.

Zou Q. and He X., "On pressure and velocity boundary conditions for the lattice Boltzmann BGK model”, Phys. Fluids, vol. 9, n 6, 1997.

Received: 19 June 2008 Accepted: 18 November 2008 